\title{
A Quality Improvement Project to Improve Evidence-Based Inhaled Nitric Oxide Use
}

\author{
Colleen A Hughes Driscoll MD, Natalie L Davis MD, Megan Miles MD, and \\ Dina El-Metwally MD PhD
}

\begin{abstract}
BACKGROUND: Inhaled nitric oxide (INO) reduces extracorporeal membrane oxygenation (ECMO) use in term and near-term neonates with persistent pulmonary hypertension of the newborn; however, its overutilization is increasing. We hypothesized that implementing a shared baseline protocol would safely improve evidence-based INO use in a Level IV neonatal ICU. METHODS: Through several plan-do-study-act cycles, a shared baseline protocol for initiation and weaning of INO was developed and implemented starting in August 2014. Based on user feedback, the shared baseline protocol was amended and re-evaluated at regular intervals. Significant changes for process and outcome measures related to utilization of INO were detected using statistical process control, bivariate analyses using $t$ test or nonparametric Wilcoxon rank-sum test as appropriate, and chi-square and Fisher exact testing as appropriate. Comparisons between the pre-plan-do-study-act group (January 2012 to July 2014) and post-plan-do-study-act group (August 2014 to October 2015) were made. RESULTS: One hundred sixteen INO courses in 95 subjects were administered during the pre-plan-do-study-act period, and 44 episodes were initiated in 39 subjects during the post-plan-do-study-act period. Process control charts demonstrate significant reductions in the percentage of INO doses $>20 \mathrm{ppm}$ and the percentage of prolonged ( $>4-d)$ INO courses. Prolonged INO courses decreased from 67.9 to $40 \%(P=.032)$, whereas the median duration of INO per course decreased from 8 to $4 \mathrm{~d}(P<.001)$. The percentage of INO courses that exceeded the dose of $20 \mathrm{ppm}$ decreased from 18.1 to $2.3 \%(P=.009)$. Very delayed INO weaning (weaning at $\left.\mathrm{F}_{\mathrm{IO}_{2}} \leq 0.40\right)$ decreased from 41.9 to $21.2 \%(P=.038)$. There were no differences in the percentage of INO courses administered to non-sedated subjects or the percentage of INO courses administered to preterm infants. There was no difference for death or ECMO between groups. CONCLUSIONS: Implementation of a shared baseline protocol to encourage appropriate INO initiation and weaning safely decreased INO exposures. Focused efforts on reducing unapproved INO use in preterm infants are warranted. Key words: inhaled nitric oxide; infant; newborn; premature; quality improvement; pulmonary hypertension; evidence-based health care. [Respir Care 2018;63(1):20-27. @ 2018 Daedalus Enterprises]
\end{abstract}

Introduction

Inhaled nitric oxide (INO) is a rapid and potent vasodilator that can lower pulmonary vascular resistance in

The authors are affiliated with the University of Maryland School of Medicine, Baltimore, Maryland. Dr El-Metwally is also affiliated with the Pediatrics Department, Faculty of Medicine, Suez Canal University, Ismailia, Egypt.

Dr Hughes Driscoll presented a version of this paper at the Mercy Hospital Pediatric Grand Rounds, held in May 1, 2015, in Baltimore, Maryland and at the University of Maryland Respiratory Care Symposium, held in March 18, 2016, in Baltimore, Maryland. patients with persistent pulmonary hypertension of the newborn. ${ }^{1}$ Since the 1990 s INO has been shown to reduce the

\footnotetext{
The authors have disclosed no conflicts of interest.

Supplementary material related to this paper is available at http:// www.rcjournal.com.

Correspondence: Colleen A Hughes Driscoll MD, 110 S Paca Street, 8th Floor, Baltimore, MD 21201. Tel.: 410-328-6003; E-mail: cdriscoll@peds.umaryland.edu.
}

DOI: $10.4187 /$ respcare.05619 
need for extracorporeal membrane oxygenation (ECMO) in term and near-term infants suffering from persistent pulmonary hypertension of the newborn and hypoxic respiratory failure, which remains the only indication for use by the FDA..$^{2-4}$ Whereas data from randomized controlled trials have demonstrated clear benefits of INO use in this population, use has increasingly extended to pediatric populations for whom studies have shown minimal evidence to support usage. Further, benefits in such populations are not well-documented in randomized controlled trials.5-9 Most notably, the use of INO in preterm infants and newborns with congenital diaphragmatic hernia has steadily risen despite recommendations to limit its use by professional groups due to insufficient and conflicting evidence. ${ }^{10-13}$ Additionally, studies have shown significant intra- and interhospital practice variation with regard to INO use in the neonatal population, suggesting deviation from evidence-based practice. ${ }^{14-16}$ Undoubtedly, the overuse and misuse of INO has become a source of unjustified health-care cost that has increased in frequency in recent years. ${ }^{5,13,17}$

At our study institution, off-label INO use, primarily among preterm infants, was found to be high. Deviations from the evidence-based management of persistent pulmonary hypertension of the newborn were noted when treating most patients, regardless of gestational age. We hypothesized that we could improve evidence-based INO use in our neonatal ICU (NICU) population while achieving 4 quality goals: (1) reducing INO exposure in non-sedated patients, (2) reducing delayed INO weaning, (3) eliminating doses of INO that are $>20 \mathrm{ppm}$, and (4) reducing prolonged INO courses.

\section{Methods}

\section{Setting}

We performed this study in the NICU of a Level IV academic teaching hospital in the mid-Atlantic region. The hospital supports a high-risk birthing center and a neonatal transport service. Twelve to 17 board-certified neonatologists supervise pediatric residents, neonatal fellows, neonatal nurse practitioners, nurses, and respiratory therapists, who care for a maximum of 52 patients. Neonatal patients requiring $\mathrm{ECMO}$ for management of persistent pulmonary hypertension of the newborn are transferred to the hospital's pediatric ICU.

\section{Study Subjects}

We included newborns and infants who received INO at any time during their NICU admission. INO courses initiated as part of anesthesia for operative procedures were excluded.

\section{QUICK LOOK}

\section{Current knowledge}

The unapproved use of inhaled nitric oxide has steadily increased, especially within specific populations, such as preterm infants. Inefficiencies and misuse of nitric oxide in the neonatal and pediatric population have been described. Improving evidence-based inhaled nitric oxide use through protocol-based quality improvement initiatives has been challenging and has often failed to result in substantial reductions in nitric oxide exposures.

\section{What this paper contributes to our knowledge}

Through the use of a shared baseline protocol, significant reductions in nitric oxide exposure times and excessive doses were demonstrated, whereas nitric oxide weaning occurred earlier.

\section{Plan-Do-Study-Act Cycles}

A project team retrospectively reviewed INO courses administered in 2012 and early 2013 to evaluate the management of pulmonary hypertension compared with evidence-based practice. ${ }^{18}$ Several areas of improvement were identified. Patients were often administered INO before other interventions to manage their pulmonary hypertension (eg, sedation) were optimized. Doses of INO frequently exceeded $20 \mathrm{ppm}$, despite a lack of clinical improvement. Initiation of INO weaning was often delayed, with patients still receiving INO despite breathing $\mathrm{F}_{\mathrm{IO}_{2}}$ as low as 0.21 . Weaning strategies were variable, and the durations of courses were lengthy, as high as $77 \mathrm{~d}$. A large portion of patients were preterm, with $65 \%$ of all INO courses being administered to patients born $<34$ weeks gestation.

Based on these observations, stretch goals were developed regarding key process measures in the management of persistent pulmonary hypertension of the newborn: (1) reducing the percentage of times that INO is initiated in non-sedated infants, (2) reducing the percentage of times that INO weaning was delayed (INO weaning that began when $\mathrm{F}_{\mathrm{IO}_{2}}$ was $<0.60$ ), (3) eliminating doses of INO that are $>20 \mathrm{ppm}$, (4) reducing the percentage of prolonged $(>4-d)$ INO courses.

Plan-Do-Study-Act Cycle 1: Staff Awareness, August to October 2014. A workshop was held to educate physicians on evidence-based management of persistent pulmonary hypertension of the newborn, FDA indications for INO, national data on INO use in preterm infants, and 
institutional data on INO use and to introduce the aims and mission of the quality improvement project. This education was provided to the neonatal nurse practitioners and respiratory therapists during staff meetings. Throughout the staff awareness phase, institutional data and project information were posted in general workspaces in the NICU and staff offices. A unit-based guideline for utilizing INO was developed during this cycle to serve as the basis for a shared baseline protocol (see Plan-Do-Study-Act Cycle 2).

Plan-Do-Study-Act Cycle 2: Implementation of Guidelines and Creation of Shared Baseline Protocol, October 2014 to July 2015. To address the areas of improvement in persistent pulmonary hypertension of the newborn management, a guideline for the initiation, administration, and weaning of INO was implemented. The guideline was designed to include evidence-based recommendations. When evidence was not available, the guideline was formed by consensus of the project team. ${ }^{18,19}$ Clinicians who ordered INO were provided a paper copy of the guideline for reference. The guideline was posted at the infant's bedside while the infant was receiving INO to guide nurses with adjusting $\mathrm{F}_{\mathrm{IO}_{2}}$ and to empower respiratory therapists to suggest INO weaning. Feedback from clinicians on acceptability and utility of the guidelines was ascertained at serial intervals. Based on feedback, the guideline was modified as long as changes were not expected to cause patient harm. These modifications led to the development of a shared baseline protocol (see online supplementary material at http://www.rcjournal.com). Prospectively collected data on INO use was posted in NICU workspaces during this period.

Plan-Do-Study-Act Cycle 3: Resident Education, May 2015 to October 2015. With as many as one third of patients in our NICU being treated by supervised resident physicians, residents were identified as stakeholders in the management of neonates with persistent pulmonary hypertension of the newborn. However, resident education had not been included in our staff awareness cycle. To improve their awareness, residents were given a 30-min lecture on the management of pulmonary hypertension, the use of INO, and an introduction to the quality improvement project during their NICU rotation.

\section{Measurements}

Pre-plan-do-study-act data were collected retrospectively. Post-plan-do-study-act data (starting in August 2014) were collected prospectively, allowing outcome data to be shared with staff at regular intervals. Outcome measurements were the duration of INO courses and the percentage of INO courses administered to preterm infants.
Process measurements were: (1) the percentage of times INO was initiated in a non-sedated infant, (2) the percentage of times INO weaning was delayed (INO weaning that began when $\mathrm{F}_{\mathrm{IO}_{2}}$ was $<0.60$ ), (3) the percentage of times that INO doses exceeded $20 \mathrm{ppm}$, and (4) the percentage of prolonged ( $>4-d)$ INO courses. Countermeasurements included length of stay and the percentage of infants who died or received ECMO.

\section{Analysis}

Quarterly data on process measurements were analyzed using statistical process control. Data were plotted on process control charts with averages and control limits reset as described by Amin. ${ }^{20}$ We performed bivariate analyses using a $t$ test or nonparametric Wilcoxon rank-sum test as appropriate for normally versus not normally distributed data. We used chi-square and Fisher exact testing as appropriate for binary and categorical variables. Statistical analyses were performed using SAS 9.3 (SAS Institute, Carey, North Carolina).

\section{Ethics}

This project was considered exempt by the University of Maryland institutional review board.

\section{Results}

One hundred sixty INO courses were administered during the study period; 95 subjects received 116 courses in the pre-plan-do-study-act period (January 2012 to July 2014), and 39 subjects received 44 courses in the postplan-do-study-act period (August 2014 to October 2015). Thirty-eight of 95 (40\%) exposed subjects from the preplan-do-study-act period were $\geq 34$ weeks gestation, whereas 20 of $39(51 \%)$ exposed subjects from the postplan-do-study-act period were $\geq 34$ weeks gestation $(P=.23)$.

We found that the frequency of doses $>20$ ppm was significantly reduced post-plan-do-study-act from 18.1 to $2.3 \%(P=.009)$, as was the mean maximal INO dose (Table 1). There was a significant reduction in prolonged INO courses from 68 to $40 \%(P=.003)$. The duration of INO courses declined by half from a median (interquartile range) of $8(3-13) \mathrm{d}$ in the pre-plan-do-study-act cohort to $4(1-7) \mathrm{d}$ in the post-plan-do-study-act cohort $(P<.001)$. We noted a decrease in the percentage of times that INO was administered in non-sedated subjects from 25 to $14.3 \%$, although this was not statistically significant. Delayed INO weaning was unchanged between the pre- and post-plando-study-act periods (Table 1). However, very delayed INO weaning, defined as $\mathrm{F}_{\mathrm{IO}_{2}} \leq 0.40$ at the initiation of INO weaning, was significantly reduced. The mean $\mathrm{F}_{\mathrm{IO}_{2}}$ at 
Table 1. Pre- and Post-Intervention Comparison of Selected Outcome and Process Measures

\begin{tabular}{|c|c|c|c|}
\hline Variables & $\begin{array}{l}\text { Pre-PDSA Group } \\
(n=95 \text { Subjects } \\
\text { and } 116 \text { Episodes })\end{array}$ & $\begin{array}{l}\text { Post-PDSA Group } \\
(n=39 \text { Subjects } \\
\text { and } 44 \text { Episodes })\end{array}$ & $P$ \\
\hline \multicolumn{4}{|l|}{ No. of INO courses/subject, $n(\%)$} \\
\hline 1 & $78(82.1)$ & $37(94.9)$ & \\
\hline$\geq 2$ & $17(17.9)$ & $2(5.1)$ & .054 \\
\hline Episodes of INO initiated in non-sedated subject ( $n=112$ vs 42$), n(\%)$ & $28(25)$ & $6(14.3)$ & .15 \\
\hline Delayed weaning at $\mathrm{F}_{\mathrm{IO}_{2}}<0.60(n=86$ vs 33$), n(\%)$ & $63(73.3)$ & $27(81.8)$ & .27 \\
\hline Very delayed weaning at $\mathrm{F}_{\mathrm{IO}_{2}}<0.40(n=86$ vs 33$), n(\%)$ & $36(41.9)$ & $7(21.2)$ & .035 \\
\hline INO doses $>20$ ppm, $n(\%)$ & $21(18.1)$ & $1(2.3)$ & .009 \\
\hline Maximal INO dose, mean \pm SD ppm & $21.56 \pm 5.3$ & $19.77 \pm 2.4$ & .033 \\
\hline Prolonged INO course $>4 \mathrm{~d}$, completed in NICU ( $n=109$ vs 35 ), $n(\%)$ & $74(67.9)$ & $14(40)$ & .003 \\
\hline Duration of INO courses, median (IQR) d & $8(3-13)$ & $4(1-7)$ & $<.001$ \\
\hline INO courses administered to preterm infants, $n(\%)$ & $75(64.7)$ & $23(52.3)$ & .15 \\
\hline Total length of stay, median (IQR) d & $49(95)$ & $41.5(96)$ & .60 \\
\hline Death or ECMO, $n(\%)$ & $42(44.2)$ & $13(33.3)$ & .24 \\
\hline $\begin{array}{l}\text { PDSA = plan-do-study-act } \\
\text { INO = inhaled nitric oxide } \\
\text { ECMO = extracorporeal membrane oxygenation }\end{array}$ & & & \\
\hline
\end{tabular}

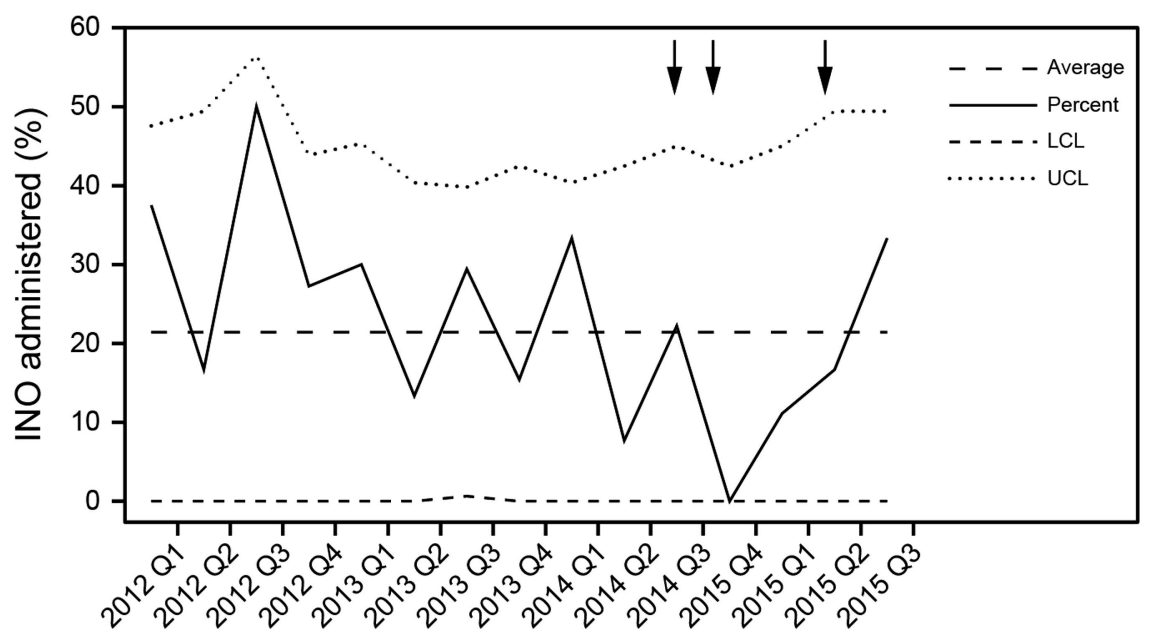

Fig. 1. Statistical process control chart showing the percentage of times that inhaled nitric oxide (INO) was administered in a non-sedated subject. LCL $=$ lower control limit, UCL $=$ upper control limit. Arrows indicate the start of plan-do-study-act cycles.

initiation of INO weaning improved from 0.44 to 0.53 , although this was not statistically significant $(P=.13)$. We observed a trend toward fewer repeat INO courses, with only 2 subjects receiving repeated courses in the post-plan-do-study-act period (Table 1). Control charts (Figs. 1-4) demonstrate quarterly data for process measures. Figures 3 and 4 show reductions of doses $>20$ ppm and of prolonged INO courses, respectively.

The percentage of all preterm infants treated at our institution who were exposed to INO during the study period was unchanged $(18.6 \%$ vs $16.2 \%, P=.42)$, as was the percentage of all courses administered to preterm infants (Table 1). The median birth gestational age of exposed infants in our cohort did increase from 29.5 to 34.1 weeks, and the percentage of subjects who were $<34$ weeks birth gestational age and were started on INO within the first month of life declined from 39.7 to $27.3 \%$, although this was not statistically significant ( $P=.15$ for both).

There was no significant difference in stay, surfactant exposure, or high-frequency ventilation use between the 2 cohorts $(P=.60, .75$, and .82 , respectively). Among term or near-term subjects with persistent pulmonary hypertension of the newborn, there was no statistically significant difference for the percentage of subjects exposed to ECMO $(P=.05)$. For the entire cohort, there was no difference in the percentage of subjects who died or were exposed to ECMO (Table 1). No subjects 


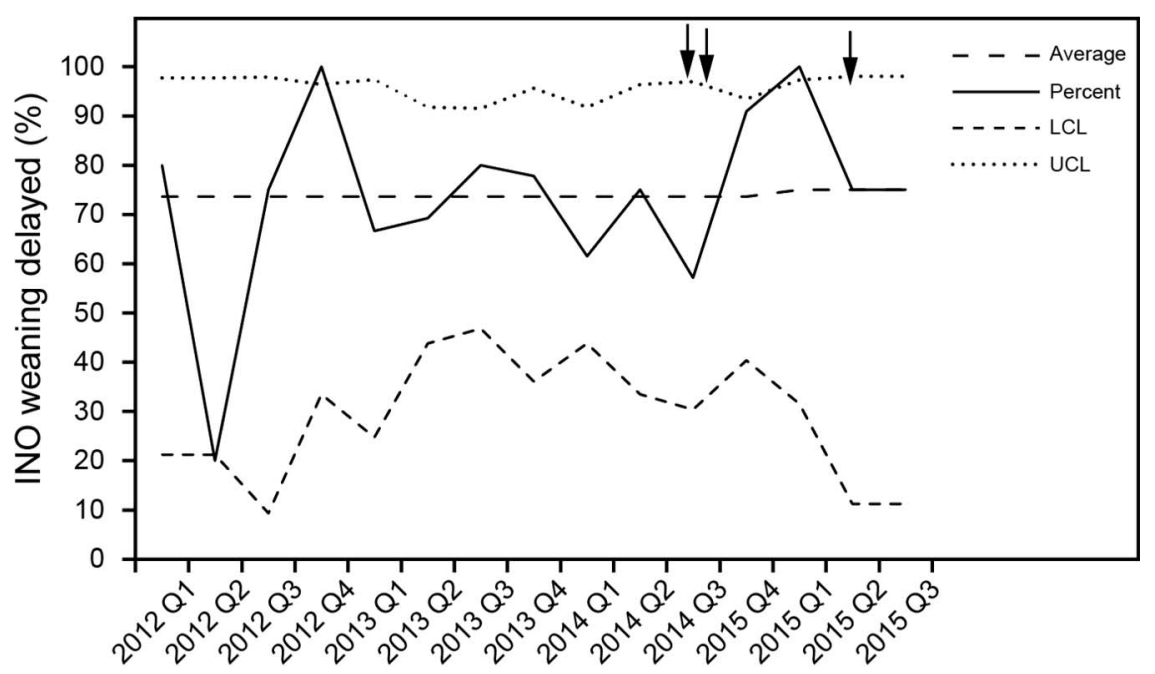

Fig. 2. Statistical process control chart showing the percentage of times inhaled nitric oxide (INO) weaning was delayed. $\mathrm{LCL}=$ lower control limit, UCL = upper control limit. Arrows indicate the start of plan-do-study-act cycles.

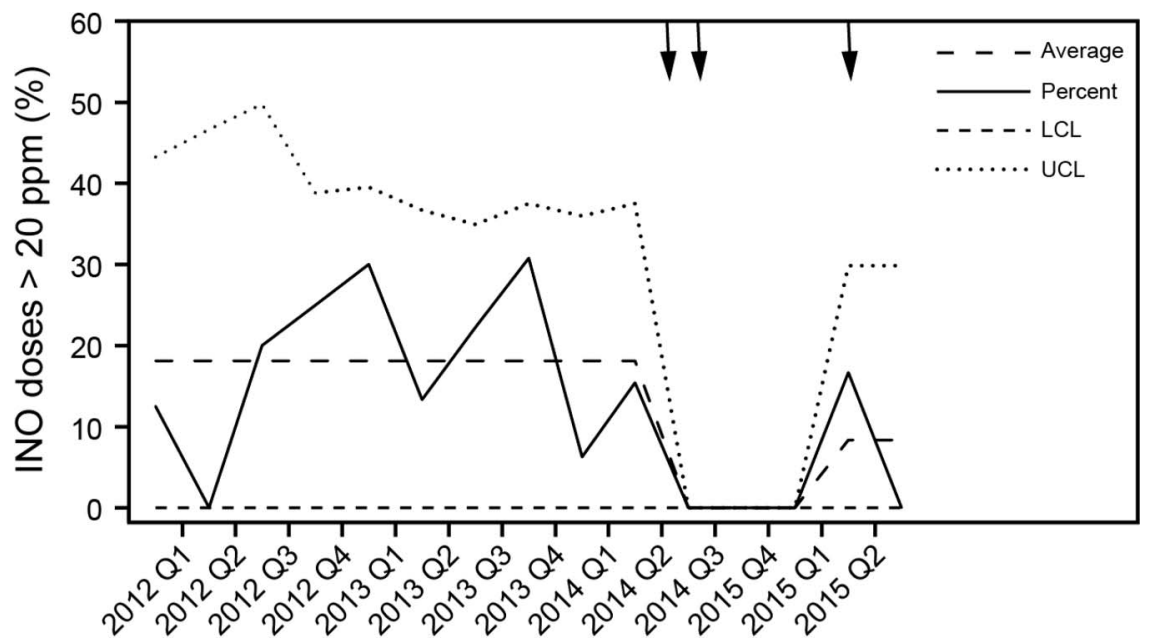

Fig. 3. Statistical process control chart showing the percentage of inhaled nitric oxide (INO) doses $>20$ ppm. LCL $=$ lower control limit, $\mathrm{UCL}=$ upper control limit. Arrows indicate the start of plan-do-study-act cycles.

in either cohort were treated with INO for prevention of bronchopulmonary dysplasia, consistent with recommended guidelines.

\section{Discussion}

Efficiency of health-care delivery is one of 6 key domains for improving health-care quality set forth by the Institute of Medicine, although perhaps one of the least measured. ${ }^{21-23}$ Waste, in the form of medication overuse and misuse, is among the factors that contribute substantially to health-care inefficiency. ${ }^{23-25}$ The implementation of practice guidelines can be a useful mechanism for directing efficient and evidence-based care. ${ }^{26}$ Our guideline, informed by a body of evidence on the management of persistent pulmonary hyper- tension of the newborn, presented an opportunity to reduce INO overuse and improve health-care efficiency. To our knowledge, this is the first quality improvement report to demonstrate a significant reduction in INO utilization by implementing a shared baseline protocol.

Following implementation, we reduced the duration of INO courses by half, suggesting more efficient drug utilization. The wide-ranging and prolonged INO duration observed at baseline led to an emphasis on INO weaning within our protocol. Simsic et al ${ }^{16}$ implemented standardized INO guidelines in a pediatric cardiothoracic ICU with similar emphasis on weaning. The authors suggest that a lack of guidance around weaning may have contributed to prolonged INO exposure during their pre-plan-do-study-act period. Interestingly, this 


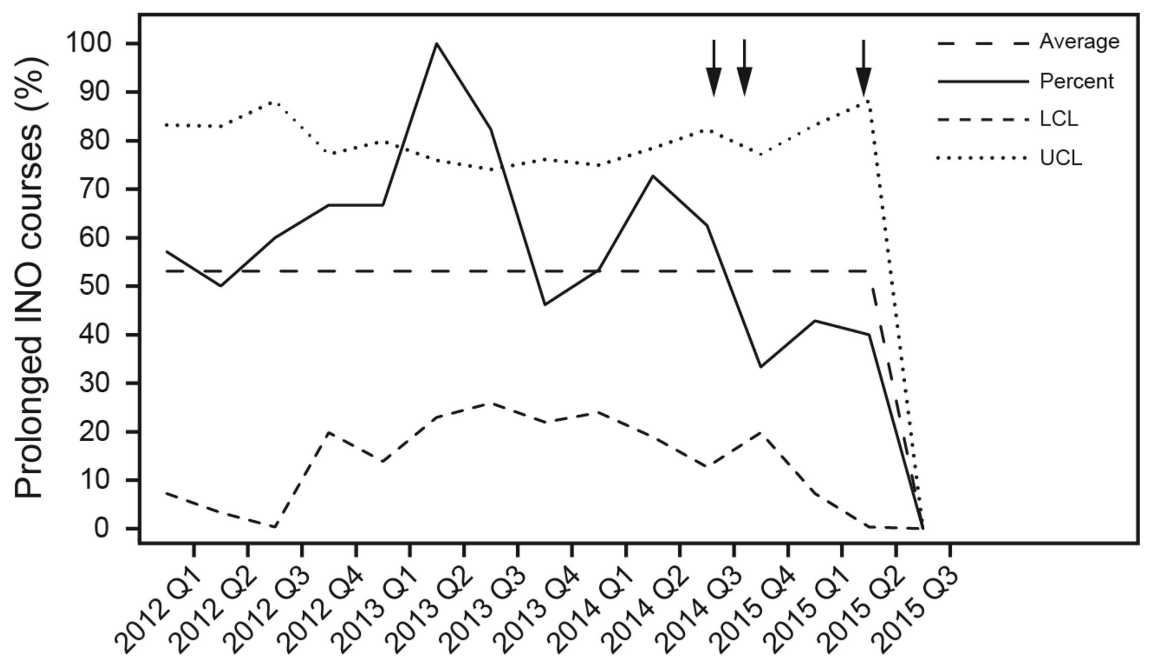

Fig. 4. Statistical process control chart showing the percentage of prolonged inhaled nitric oxide (INO) courses. LCL $=$ lower control limit, $\mathrm{UCL}=$ upper control limit. Arrows indicate the start of plan-do-study-act cycles.

did not translate into a significant reduction in utilization. Similar results were reported by Todd Tzanetos et $\mathrm{al}^{27}$ who found reductions in direct costs for INO within their pediatric ICU after protocol implementation but no significant change in duration of INO use. Explanations for the differing impact of protocol implementation among the studies are unclear, but the process of creating a shared baseline protocol may be responsible for our success. Shared baseline protocols allow for modifications based on individual patient needs, recognizing that one protocol is unlikely to adequately support the management of every patient. Shared baseline protocols create an opportunity for frequent protocol adjustments, thereby encouraging clinicians to adhere to evidence-based management, ${ }^{28}$ and may help reduce the barriers that are often associated with achieving protocol compliance among providers. Although we did not aim to reduce provider variation in management, we found that providers were active in suggesting protocol modifications. Ultimately, provider alterations may have led to reductions in utilization. For example, one modification involved removing the oxygenation index as a criterion for INO weaning. Elmekkawi et $\mathrm{al}^{29}$ reported reduced INO use at a Level IV Canadian NICU after implementing a stewardship program, which shared a protocol review and amendment strategies similar to ours. Our results reinforce the authors' conclusion that stakeholder support of guideline content is essential to efficacious implementation.

There may be a number of additional reasons for our implementation success. We note that our utilization of INO was higher at baseline (median 8 vs $2.6 \mathrm{~d} /$ event) when compared with the Simsic et al study, ${ }^{16}$ which could have provided more opportunity for easy improvement gains. Additionally, our cohort included a signif- icant number of preterm infants, a population for which INO use has been discouraged by professional groups. Although we continued to utilize INO in our preterm infants during the post-plan-do-study-act period, guidance from these professional groups may have influenced physicians to especially limit exposure times when treating preterm subjects. Our reduction in repeated INO courses, which occurred primarily among the preterm infant population, supports this assertion.

Regrettably, although the median birth gestational age increased in our post-plan-do-study-act cohort, INO use in our preterm population persists. INO use in this group is known to be significant, with marked variability among NICUs in terms of the percentage of preterm infants exposed to INO, the age at which INO is initiated, and the average number of days of exposure. ${ }^{8,14}$ Rates of exposure for preterm infants are reported as high as $19 \%$ in some centers and tend to be greatest at the earliest gestations. ${ }^{7-9,15,30}$ Preterm infants account for $46 \%$ of all patients receiving INO, making this population an important area of focus in overuse reduction. ${ }^{7}$ Following the 2010 NICHD Consensus Development Conference Statement on Inhaled Nitric Oxide Therapy for Premature Infants, ${ }^{13}$ which discourages INO use in infants $<34$ weeks gestation, the Neonatal Research Network observed a reduction in use among preterm infants within 13 representative NICUs. ${ }^{9}$ Unfortunately, this improvement was not generalized to broader NICU networks. ${ }^{7}$ Given the high cost $(\$ 105.55 / \mathrm{h}$ during our study) one might expect a reduction in usage from financial pressures alone. ${ }^{9}$ However, INO use in preterm infants may in part be related to rescue care, as Stenger et $\mathrm{al}^{14}$ showed an association between INO exposure and mortality in preterm infants. Further, the highest incidence of INO exposure is found to occur at the lowest 
gestational age and birthweight. ${ }^{8}$ The persistent overutilization, despite the cost, underscores the need for focused quality improvement interventions in preterm infants in addition to better treatment strategies for preterm infants with chronic lung disease and resultant hypoxic respiratory failure. To date, successful approaches for decreasing INO use among preterm infants have not been reported.

There are limitations to our study. Primarily, we have yet to establish that improvement gains can be sustained. However, notable practice changes with regard to INO use are evident and probably contribute to an overall culture change that will be sustainable. Ongoing surveillance of our INO usage pattern will be an important aspect of this project in the future. Second, we were unable to show statistically significant reductions in INO use among preterm infants. This may reflect the relative post-plan-do-study-act sample size, compared with the preplan-do-study-act sample. Given the trend toward increasing birth gestational age between the pre- and post-plan-do-studyact groups, we suspect that ongoing evaluation may prove an impact on the preterm population, particularly as practice changes are solidified within the culture of our NICU. Although we did not undertake a cost analysis to understand the financial implications of these improvements, based on our hospital contract rate, we estimate an average reduction in INO costs of $\$ 10,132.80$ per INO course. Finally, our approach may have been most effective because of the high degree of overuse among our providers. In units where use is not as abundant, guideline implementation may be less beneficial. It is reassuring that a nitric oxide stewardship program that shares common features with our interventions effectively reduced INO use in a more conservative setting. ${ }^{29}$

\section{Conclusions}

Shared baseline protocols have been shown to improve care delivery through implementation of evidence-based practice. The creation and implementation of a shared baseline protocol, along with supportive education, safely reduced INO overuse in the NICU setting. INO exposures among preterm infants remain high, and effective strategies to reduce exposures in this population are needed.

\section{REFERENCES}

1. Ichinose F, Roberts JD, Jr., Zapol WM. Inhaled nitric oxide: a selective pulmonary vasodilator: current uses and therapeutic potential. Circulation 2004;109(25):3106-3111.

2. Neonatal Inhaled Nitric Oxide Study Group. Inhaled nitric oxide in full-term and nearly full-term infants with hypoxic respiratory failure. N Engl J Med 1997;336(9):597-604.

3. Clark RH, Kueser TJ, Walker MW, Southgate WM, Huckaby JL, Perez JA, et al. Low-dose nitric oxide therapy for persistent pulmonary hypertension of the newborn: Clinical Inhaled Nitric Oxide Research Group. N Engl J Med 2000;342(7):469-474.
4. United States Food and Drug Administration. Drugs@FDA; FDA approved drug products. http://www.accessdata.fda.gov/scripts/cder/ daf/index.cfm?event $=$ BasicSearch.process. Accessed January 17, 2017.

5. Campbell BT, Herbst KW, Briden KE, Neff S, Ruscher KA, Hagadorn JI. Inhaled nitric oxide use in neonates with congenital diaphragmatic hernia. Pediatrics 2014;134(2):e420-e426.

6. Clark RH, Ursprung RL, Walker MW, Ellsbury DL, Spitzer AR. The changing pattern of inhaled nitric oxide use in the neonatal intensive care unit. J Perinatol 2010;30(12):800-804.

7. Ellsworth MA, Harris MN, Carey WA, Spitzer AR, Clark RH. Offlabel use of inhaled nitric oxide after release of NIH consensus statement. Pediatrics 2015;135(4):643-648.

8. Handley SC, Steinhorn RH, Hopper AO, Govindaswami B, Bhatt DR, Van Meurs KP, et al. Inhaled nitric oxide use in preterm infants in California neonatal intensive care units. J Perinatol 2016;36(8):635-639.

9. Truog WE, Nelin LD, Das A, Kendrick DE, Bell EF, Carlo WA, et al. Inhaled nitric oxide usage in preterm infants in the NICHD Neonatal Research Network: inter-site variation and propensity evaluation. J Perinatol 2014;34(11):842-846.

10. Finer NN, Barrington KJ. Nitric oxide for respiratory failure in infants born at or near term. Cochrane Database Syst Rev 2006;(4): CD000399.

11. American Academy of Pediatrics Committee on Fetus and Newborn. Use of inhaled nitric oxide. Pediatrics 2000;106(2 Pt 1):344-345.

12. Peliowski A, Canadian Pediatric Society, Fetus and Newborn Committee. Inhaled nitric oxide use in newborns. Paediatr Child Health 2012;17(2):95-100.

13. Cole FS, Alleyne C, Barks JD, Boyle RJ, Carroll JL, Dokken D, et al. NIH Consensus Development Conference statement on inhaled nitric oxide therapy for premature infants. NIH Consens State Sci Statements 2010;27(5):1-34.

14. Stenger MR, Slaughter JL, Kelleher K, Shepherd EG, Klebanoff MA, Reagan P, et al. Hospital variation in nitric oxide use for premature infants. Pediatrics 2012;129(4):e945-e951.

15. Soraisham AS, Harabor A, Shivananda S, Alvaro R, Ye XY, Lee SK, Shah PS. Trends and variations in the use of inhaled nitric oxide in preterm infants in Canadian neonatal intensive care units. Am J Perinatol 2016;33(7):715-722.

16. Simsic JM, Harrison S, Evans L, McClead R, Teske D. Reducing variation in the use of inhaled nitric oxide. Pediatrics 2014;133(6): e1753-e1758.

17. Ho T, Dukhovny D, Zupancic JA, Goldmann DA, Horbar JD, Pursley DM. Choosing wisely in newborn medicine: five opportunities to increase value. Pediatrics 2015;136(2):e482-e489.

18. Keszler M. Guidelines for rational and cost-effective use of INO therapy in term and preterm infants. J Clin Neonatol 2012;1(2):59-63.

19. Ware AM, Golombek SG. Weaning of inhaled nitric oxide: is there a best strategy? J Pediatr Neonatal Individ Med 2015;4(1):e040124. doi: $10.7363 / 040124$

20. Amin SG. Control charts 101: a guide to health care applications. Qual Manag Health Care 2001;9(3):1-27.

21. Agency for Healthcare Research and Quality. The six domains of health care quality. 2016. http://www.ahrq.gov/professionals/qualitypatient-safety/talkingquality/create/sixdomains.html\#_ftn2. Accessed June 8, 2016.

22. Institute of Medicine. Performance measurement: accelerating improvement. Washington, DC: National Academy Press; 2005.

23. Newton EH, Zazzera EA, Van Moorsel G, Sirovich BE. Undermeasuring overuse: an examination of national clinical performance measures. JAMA Intern Med 2015;175(10):1709-1711.

24. Gamble M. 8 types of waste in healthcare. Becker's Hospital Review. September 10, 2013. http://www.beckershospitalreview.com/hospital- 
management-administration/8-types-of-waste-in-healthcare.html. Accessed August 26, 2017.

25. Morgan DJ, Wright SM, Dhruva S. Update on medical overuse. JAMA Intern Med 2015;175(1):120-124.

26. Woolf SH. Practice guidelines: a new reality in medicine. I. Recent developments. Arch Intern Med 1990;150(9):1811-1818.

27. Todd Tzanetos DR, Housley JJ, Barr FE, May WL, Landers CD. Implementation of an inhaled nitric oxide protocol decreases direct cost associated with its use. Respir Care 2015;60(5):644-650.
28. James BC, Savitz LA. How Intermountain trimmed health care costs through robust quality improvement efforts. Health Aff 2011;30(6):1185-1191.

29. Elmekkawi A, More K, Shea J, Sperling C, Da Silva Z, Finelli M, et al. Impact of stewardship on inhaled nitric oxide utilization in a neonatal ICU. Hosp Pediatr 2016;6(10):607-615.

30. Padula MA, Grover TR, Brozanski B, Zaniletti I, Nelin LD, Asselin $\mathrm{JM}$, et al. Therapeutic interventions and short-term outcomes for infants with severe bronchopulmonary dysplasia born at $<32$ weeks' gestation. J Perinatol 2013;33(11):877-881.

This article is approved for Continuing Respiratory Care Education credit. For information and to obtain your CRCE

(free to AARC members) visit

www.rcjournal.com 